\title{
Das Smartphone-Dilemma und die Frage der Offenheit
}

\author{
Matthias Knoll (D)
}

Angenommen: 11. Februar 2021 / Online publiziert: 26. Februar 2021

(C) Der/die Autor(en) 2021

Dieser Einwurf ist kein Versuch, den Aspekt des gefühlt zunehmenden „Zwangs“ zur Nutzung eines Smartphones und das Problem der damit aktuell verbundenen Abhängigkeit von zwei großen Plattformen wissenschaftlich zu beleuchten, sondern dem Wunsch entsprungen, der Problematik zu mehr Sichtbarkeit (und damit vielleicht auch Prominenz?) zu verhelfen und nicht zuletzt, Erfahrungen weiterzugeben. Ein interessanter Forschungsgegenstand wäre die technisch, ökonomisch und gesellschaftspolitische Fragestellung einer vermeintlich zunehmenden Abhängigkeit von Smartphones und die daraus aktuell folgende Abhängigkeit von zwei großen Plattformen wohl in jedem Fall.

Denn es ist ein Dilemma. Das Smartphone ist aus unserem Alltag praktisch nicht mehr wegzudenken. Wir nutzen es mehr oder weniger intensiv für die unterschiedlichsten Aufgaben. Eine echte Wahl haben wir dabei jedoch im Prinzip nicht. Und das weder in Bezug darauf, ob wir es überhaupt und grundsätzlich nutzen wollen, noch, ob wir uns in den Kosmos großer, potenziell über alle Lebensbereiche Daten sammelnder Anbieter begeben wollen. Warum ist das so? Zur Beantwortung dieser Frage ist zunächst ein Blick auf die grundsätzliche Entscheidung des Für oder Wider der Nutzung dieser Geräte hilfreich.

Manche von uns hassen das Smartphone, weil sie generell gerne darauf verzichten würden, sie aber zunehmend keine Alternative zur Nutzung mehr haben. Beispielsweise, wenn Kreditkartenautorisierungen erfolgen müssen, die Karten-ausgebende Bank jedoch seit kurzem keine andere Autorisierungsform als über eine App anbietet. Oder wenn der Betrieb von ,smarten Geräten“ ausschließlich in Verbindung mit einer Hersteller-App und unter Nutzung von Cloud-Diensten möglich ist. Dann muss man zum Smartphone greifen, ob man will oder nicht. Solche Zwänge werden

M. Knoll (四)

Hochschule Darmstadt, Darmstadt, Deutschland

E-Mail: matthias.knoll@h-da.de 
zunehmen, es ist lediglich eine Frage der Zeit, bis immer mehr Unternehmen ihre Dienstleistungsangebote vollständig auf die mobilen Assistenten umstellen oder über ihre Nutzung vielfältige, attraktive Vorteile einräumen und damit indirekt all jene benachteiligen, die sich der Nutzung verschließen.

Doch warum verschließen sich Menschen vor dieser Technologie? Ein Grund könnte die Sorge im Kontext des Umgangs mit persönlichen Daten sein. Hardware und Systemsoftware in einem Smartphone ermöglichen die umfassende Erkundung von Gewohnheiten, Eigenschaften und Verhaltensweisen. Leistungsfähige Sensorik kann alles von Interesse detektieren, insbesondere, wenn das Smartphone durch entsprechende Wearables (etwa die Smartwatch oder ein Fitness-Tracker) funktional erweitert wird. Unabhängig davon, ob das nun tatsächlich geschieht oder nicht. Niemand weiß mit Sicherheit zu sagen, wer wann wozu welche Daten erhebt, speichert, analysiert und daraus Kapital schlägt. Oder was passiert, wenn diese mehr oder weniger sensiblen Daten Opfer eines Angriffs werden und in der Folge frei im Internet zugänglich sind. Ein anderer Grund könnte sein, dass Menschen einer durch ein Duopol entstehenden Marktmacht prinzipiell kritisch gegenüberstehen. Im September 2020 dominierten die beiden Anbieter Apple und Google mit 99,42\% den Markt ${ }^{1}$. Das war nicht immer so, aber das ist wieder eine andere Geschichte. Weitere $0,32 \%$ teilen sich vier altbekannte Unternehmen, die versuchen, sich gegen die Übermacht zu stemmen. Die verbleibenden $0,26 \%$ entfallen auf undokumentierte Alternativen, darunter zahlreiche freie Lösungen, unter anderem auf Debian-Basis und auf Basis der Android Mobile Platform (dann i.d.R. ohne Google-Dienste).

Aber auch diejenigen Nutzer, die ihr Smartphone grundsätzlich lieben und es für überaus praktisch halten, weil es für nahezu jede Aufgaben- oder Fragestellung eine helfende App gibt, haben aufgrund dieses Duopols keine echte Wahlmöglichkeit. Ob bei der Suche nach der nächstmöglichen Bus- oder Bahnverbindung, bei mobilen Bezahlvorgängen, als Unterstützung beim Backen oder Kochen oder sogar beim Sex: Die wenigsten sogenannten nativen Apps gibt es für andere als die beiden dominierenden Plattformen, zum Teil auch deshalb, weil sie so programmiert sind, dass sie bestimmte Dienste der beiden großen Anbieter zwingend benötigen. Zudem ist es ökonomisch sofort verständlich, einen Fokus zu setzen, weil es wenig sinnvoll wäre, Zeit, Geld und Entwicklungs-Know-how in eine App zu stecken, deren Nutzung sich auf wenige hundert Installationen beschränken würde. Wenn wir also die vielen Annehmlichkeiten nutzen wollen, müssen wir uns aktuell de-facto für eine der beiden Welten, mitunter aus „Kompatibilitätsgründen“ sogar für ein bestimmtes Modell entscheiden.

Was fehlt ist Offenheit! Offenheit bedeutet in diesem Kontext, dass Apps nicht zwingend an einen App-Store und damit an eine technische Plattform gebunden sind, dass auch alternative Dienste (für Standort-Informationen etwa Openstreetmap) genutzt werden können, dass alle über die Verwendung ihrer Daten in diesen Apps jederzeit vollständig und vor allem ohne Funktionseinbußen entscheiden können und dass Apps, zumindest aber das Betriebssystem und gegebenenfalls auch die Hardware nicht nur in Teilen, sondern vollständig Open Source sind.

\footnotetext{
${ }^{1}$ Vgl. https://de.statista.com/statistik/daten/studie/184332/umfrage/marktanteil-der-mobilen-betriebssyste me-in-deutschland-seit-2009/.
} 
Mit Blick auf eine App-Vielfalt für alternative Plattformen scheint vielleicht Licht am Ende des Tunnels möglich: Sogenannte Progressive Web Apps (PWA) ermöglichen trotz Zugriff über den Browser ein Nutzungserlebnis das mit kleineren Abstrichen mit nativen Apps annähernd vergleichbar ist. Sie böten daher in vielen Fällen vielleicht eine elegante Möglichkeit, sich von den beiden dominierenden Plattformen und deren App Stores zu lösen. Vieles spräche deshalb dafür, dass Unternehmen für ihre mobilen Angebote eine PWA-Strategie entwickeln².

Anders sah es bislang auf Hardware- und Systemsoftware-Seite aus. Zwar ist ein großer Teil des Betriebssystems Android Open-Source-Software, doch können viele Funktionalitäten nur mit proprietären Code-Erweiterungen genutzt werden. Zudem muss - technisch bedingt - ein kompliziertes Customizing des „StandardAndroid“" auf das jeweilige Endgerät erfolgen, was langfristig Updates zu einem betriebswirtschaftlichen Abenteuer werden lässt und/oder in ein selbstausbeuterisches Entwickler-Leben führt.

Der mühevolle Weg, für diese Fragen (bestmögliche) Lösungen mit all ihren Limitationen zu finden, fasst Urs Pfister in einem Beitrag vom April 2020 treffend zusammen ${ }^{3}$. Auf Basis seiner umfassenden Erfahrungen mit „freiem“ Android und seinen Überlegungen zu Linux-Alternativen lassen sich wichtige Anforderungen an ein tatsächlich freies Smartphone zusammenfassen. Es beginnt damit, dass ein freies System sich beliebig anpassen und lokal sichern lassen muss. Zudem müssen Apps ohne die zwangsweise Bindung an proprietäre Dienste sowie ohne Anmeldung und Geräteverknüpfung betrieben werden können. Im Idealfall sind auch die für den Betrieb der Geräte zwingend notwendigen zahlreichen Firmware-Dateien Open-Source. Und der Zugriff auf die Cloud muss frei wählbar sein und nicht zum (unveränderlichen) Standard gehören. Eine mögliche Lösung für die Jahre 2021 und danach oder doch zumindest ein Impulsgeber könnte vielleicht ein rein Linux-basiertes Smartphone sein, dass sich dem sprichwörtlichen Kampf David gegen Goliath stellen kann.

Genau daran wurde im vergangenen Jahr und bereits zuvor intensiv gearbeitet.

Welche Optionen ergeben sich für „Normalnutzer“ mit einer gewissen TechnikAffinität aktuell? Denn Alternativen haben nur dann eine Chance, wenn sie ohne umfassendes Hintergrundwissen praktisch ,out-of-the-box“ eingesetzt werden können, und wenn sie im Alltag funktional (nahezu) gleichwertig zu bisherigen Angebote sind - insbesondere, was zentrale Apps wie Browser, E-Mail, Chat, Kamera/ Mikrofon-Nutzung und die Nutzung von Geoinformation (Standort/Kartendienste) angeht.

In diesem Zusammenhang wird vielfach Ubuntu Touch als mobiles Betriebssystem genannt. Es ist der von der Ubports Stiftung und deren Community mitgetragene mobile „Ableger“ des Debian-Derivats Ubuntu und ermöglicht aus diesem Grund den prinzipiellen Zugang zu den meisten Linux-basierten Apps. Zwar gibt es eine Reihe weiterer Systeme, sie sind jedoch hinsichtlich ihrer Verbreitung und/oder ihrer „Usability“ nicht vergleichbar, erfordern sie doch vielfach auf Nutzerseite um-

\footnotetext{
2 Vgl. https://www.new-communication.de/neues/detail/10-gruende-fuer-progressive-web-apps/.

3 Vgl. https://www.pro-linux.de/artikel/2/1995/frei-mit-android-drei-jahre-open-source-smartphone. html.
} 
fassendes Informatik-Know-how. Einzige Ausnahme ist das Betriebssystem PureOS des kalifornischen Anbieters purism, das ebenfalls auf Debian basiert und das auch bei allen weiteren purism-Produkten genutzt wird. Die generelle Verfügbarkeit von Apps könnte daher vergleichbar gut sein, doch noch ist das nicht klar.

Zwar gab es in der Vergangenheit immer wieder Endgeräte, die mit Ubuntu Touch ausgerüstet waren. Häufig jedoch boten sie wichtige Eigenschaften wie LTE nicht, als diese in den marktüblichen Geräten längst Standard waren. Das lag unter anderem auch daran, dass in der Regel lediglich ältere, von den Herstellern nicht mehr unmittelbar im technischen und betriebswirtschaftlichen Fokus stehende, meist leistungsschwächere Geräte dafür genutzt werden konnten. Bei neueren Modellen war der Entwicklungsgemeinde für die Treiber-Entwicklung der in gleicher Weise auch für das Android-Customizing notwendige Zugang zum inneren Aufbau der Endgeräte aus vielerlei - ökonomisch und marktpolitisch nachvollziehbaren - Gründen verwehrt. Für die alltägliche Nutzung und insbesondere als vollwertiger Ersatz für das ,typische“ Smartphone waren diese bislang erhältlichen Geräte daher nicht einsetzbar.

Anfang 2021 werden nun insgesamt drei neu entwickelte bzw. angepasste Geräte angeboten: Das im Frühjahr 2020 gestartete und seither mit zahlreichen Updates versorgte PinePhone, das von Pine Microsystems und der großen Pine64-Community entwickelt wurde sowie das in Deutschland koordinierte und produzierte VollaPhone der Hallo Welt Systeme auf Basis eines angepassten Gigaset GS290. Beide können mit Ubuntu Touch betrieben werden. Das dritte Gerät ist das Librem 5 des bereits erwähnten amerikanischen Herstellers purism mit dem Debian-basierten $\mathrm{Pu}-$ reOS. Die ersten beiden Geräte wurden Ende November/Anfang Dezember bereits in größeren Stückzahlen in Deutschland ausgeliefert, die Auslieferung des Librem 5 hat zum Jahreswechsel begonnen, noch sind jedoch nicht alle Vorbestellungen bei ihren Empfängern angekommen.

Zudem gibt es zahlreiche Projekte mit weiteren Linux-basierten Betriebssystemen für eine Reihe von älteren und neueren Geräten, einige interessante HardwareEntwicklungen laufen aktuell ${ }^{4}$. Wer sich mit dem Thema befassen möchte, muss jedoch fundiertes Grundwissen mitbringen oder sich einarbeiten. Die Installation alternativer Betriebssysteme auf ursprünglich nicht dafür vorgesehenen Geräten ist möglich, aber nicht immer einfach, wie auch Urs Pfister in seinem oben zitierten Beitrag darstellt.

Konzentriert man sich auf die neu verfügbaren Linux-Smartphones, ist das PinePhone das preisgünstigste Gerät, das Librem 5 das teuerste, das Volla-Phone liegt dazwischen. Hinsichtlich Abmessungen, Gewicht und Optik liegen die Geräte dicht beieinander. Manches High-End-Smartphone mag dünner und leichter sein, das entscheidende Kriterium hier ist jedoch die Alltagstauglichkeit verbunden mit der Tatsache, dass ein alternatives und freies Betriebssystem zur Verfügung steht. Mit Blick auf eine unmittelbare Nutzung im Alltag auch durch wenig erfahrene Anwender schneidet aktuell das Volla-Phone gegenüber dem PinePhone besser ab, alle Grundfunktionalitäten stehen stabil zur Verfügung. Ein zentraler Vorteil des PinePhone ist hingegen, dass alle Teile leicht für Experimente getauscht werden können, ein-

\footnotetext{
${ }^{4}$ Eine sehr gute und übersichtliche Informationsquelle hierfür ist https://tuxphones.com.
} 
schließlich des Mainbords. Damit erschließt das PinePhone eine große experimentelle Welt. Über ein Update der Mutterplatine ist es in der Lage, bis zu 13 verschiedene Betriebssysteme zu booten, es lässt sich also leicht herausfinden, welche Distribution das Beste aus der Hardware herauszuholen vermag oder die beste „Usability“ aufweist. Wer technisch interessiert ist und sich tiefer einarbeiten möchte, findet im PinePhone das ideale Gerät.

Wie sich das Librem 5 dazu positionieren kann, bleibt abzuwarten. Bis Redaktionsschluss lag das Gerät leider noch nicht vor.

Doch damit sich Linux-Smartphones tatsächlich durchsetzen zu können, braucht es die Unterstützung möglichst vieler Organisationen aus allen Branchen. Sei es, indem sie eine PWA-Strategie verfolgen, die es ermöglichen, Apps ohne Zusatzaufwände für Anbieter und Nutzende und ohne funktionale Einbußen auch abseits der beiden großen App-Stores einzusetzen. Oder sei es, dass sie aus Marketing-, Imageoder anderen Gründen (vielleicht gar heimliches „Rebellentum“) auch für diese Plattform - Community-unterstützt - native Apps bereitstellen und so - wie bereits im Desktop- und Server-Markt in naher Zukunft eine echte Alternative schaffen, bei deren Nutzung die volle Kontrolle über die eigenen Daten sichergestellt ist.

Es bleibt spannend.

Funding Open Access funding enabled and organized by Projekt DEAL.

Open Access Dieser Artikel wird unter der Creative Commons Namensnennung 4.0 International Lizenz veröffentlicht, welche die Nutzung, Vervielfältigung, Bearbeitung, Verbreitung und Wiedergabe in jeglichem Medium und Format erlaubt, sofern Sie den/die ursprünglichen Autor(en) und die Quelle ordnungsgemäß nennen, einen Link zur Creative Commons Lizenz beifügen und angeben, ob Änderungen vorgenommen wurden.

Die in diesem Artikel enthaltenen Bilder und sonstiges Drittmaterial unterliegen ebenfalls der genannten Creative Commons Lizenz, sofern sich aus der Abbildungslegende nichts anderes ergibt. Sofern das betreffende Material nicht unter der genannten Creative Commons Lizenz steht und die betreffende Handlung nicht nach gesetzlichen Vorschriften erlaubt ist, ist für die oben aufgeführten Weiterverwendungen des Materials die Einwilligung des jeweiligen Rechteinhabers einzuholen.

Weitere Details zur Lizenz entnehmen Sie bitte der Lizenzinformation auf http://creativecommons.org/ licenses/by/4.0/deed.de. 